\title{
Characterization of quadratic growth of extended-real-valued functions
}

\author{
Jin jiang Wang ${ }^{1,2}$ and Wen Song ${ }^{1,3^{*}}$
}

${ }^{\text {"Correspondence: }}$

wsong@hrbnu.edu.cn

${ }^{1}$ Department of Mathematics, Harbin Institute of Technology,

Harbin, 150001, China

${ }^{3}$ School of Mathematical Sciences,

Harbin Normal University, Harbin,

150025, China

Full list of author information is

available at the end of the article

\begin{abstract}
This paper shows that the sharpest possible bound in the second-order growth condition of a proper lower semicontinuous function can be attained under some assumptions. We also establish a relationship among strong metric subregularity, quadratic growth, the positive-definiteness property of the second-order subdifferential/generalized Hessian, the strong metric regularity, and tilt stability in a finite-dimensional setting.
\end{abstract}

MSC: 49J53; 14P10; 54C60; 65K10

Keywords: quadratic growth; Mordukhovich (limiting) subdifferential; strong metric regularity; strong metric subregularity; prox-regular

\section{Introduction}

This work is devoted to studying the relationships among strong metric subregularity, the quadratic growth condition, strong metric regularity, and the positive-definiteness property of the second-order subdifferential/generalized Hessian in finite and infinitedimensional settings.

It is well known in nonlinear optimization that the quadratic growth conditions of extended-valued functions play a central role (see, e.g., [1-3]). In a nonsmooth setting, various metric regularity properties of the subdifferential of a lower semicontinuous convex function acting in a Hilbert space were established by Aragón Artacho and Geoffroy [4] in terms of the quadratic growth condition. Later, motivated by some results in [5], Aragón Artacho and Geoffroy [6] extended their characterizations from [4] to convex functions defined on arbitrary Banach spaces and pointed out that if the subgradient mapping $\partial f$ of a proper l.s.c. convex function $f$ defined on a Banach space is strongly metrically subregular at $\left(\bar{x}, \bar{x}^{*}\right)$ with modulus $\kappa>0$, then the following quadratic growth condition holds:

$$
f(x) \geq f(\bar{x})+\left\langle\bar{x}^{*}, x-\bar{x}\right\rangle+\frac{c}{2}\|x-\bar{x}\|^{2} \quad \text { for all } x \in U,
$$

where $c \in\left(0, \frac{1}{2 \kappa}\right)$ and $U$ is a neighborhood of $\bar{x}$. They raised the question whether the bound of the constant in the quadratic growth condition (1) may be improved (see [6], Remark 3.2). Drusvyatskiy et al. [7] extended the characterizations from [6] to proper lower semicontinuous functions defined on Asplund spaces and gave an affirmative answer to the above question via showing that the constant $c$ in (1) can be chosen arbitrarily in $\left(0, \frac{1}{\kappa}\right)$.

(c) 2016 Wang and Song. This article is distributed under the terms of the Creative Commons Attribution 4.0 International License (http://creativecommons.org/licenses/by/4.0/), which permits unrestricted use, distribution, and reproduction in any medium, provided you give appropriate credit to the original author(s) and the source, provide a link to the Creative Commons license, and indicate if changes were made. 
In other lines of development, in [8], the second-order subdifferential/generalized Hessian is used to characterize the tilt-stable local minimizers introduced by Poliquin and Rockafellar [8] for prox-regular and subdifferentially continuous functions on $\mathbb{R}^{n}$. Later, Mordukhovich and Nghia [5] extended this characterization to the settings of Hilbert spaces by using a new notion of the combined second-order subdifferential. Quite recently, Drusvyatskiy et al. [7] extended the known equivalences between metric regularity and the strong metric regularity properties of the gradient mappings for $\mathcal{C}^{2}$-smooth functions, characterized via the classical Hessian, to the general class of prox-regular and subdifferentially continuous functions on $\mathbb{R}^{n}$ in terms of the second-order subdifferential/generalized Hessian.

The current work aims to achieve new results in the aforementioned directions by improving the known characterizations. After recording in Section 2 the necessary definitions and facts that will be needed throughout the manuscript, we devote Section 3 to characterizations of the second-order growth condition of a proper lower semicontinuous function. Theorem 3.1 provides a sufficient condition which allows strong metric subregularity with modulus $\kappa$ to imply the second-order growth condition with the constant $c=1 / \kappa$. Theorem 3.2 gives a sufficient condition for metric subregularity in a nonconvex setting. In Section 4, in finite-dimensional setting, we establish an equivalence among strong metric subregularity, quadratic growth, the positive-definiteness property of the second-order subdifferential/generalized Hessian, the strong metric regularity and tilt stability, and we show that the constant $c$ in the quadratic growth condition can attain $1 / \kappa$ (Theorem 4.2).

\section{Preliminaries}

In this section, we summarize some of the fundamental tools used in variational analysis and nonsmooth optimization. Readers can refer [9-12] for more details. Throughout this paper, unless otherwise stated, $X$ and $Y$ are Banach spaces. The symbol $\rightarrow$ always denotes the convergence relative to the norm while the symbol $\stackrel{w^{*}}{\rightarrow}$ signifies the weak* convergence in the dual space $X^{*}$. The closed ball centered at $x \in X$ of radius $r$ is denoted by $\mathbb{B}_{r}(x)$. We denote by $\mathbb{R}_{--}$the set $\{x \in \mathbb{R} \mid x<0\}$. The distance function associated with a nonempty set $\Omega \in X$ is defined by

$$
d(x ; \Omega):=\inf _{y \in \Omega}\|x-y\|, \quad x \in X
$$

Let $F: X \rightrightarrows Y$ be a set-valued mapping between Banach spaces $X$ and $Y$. The domain and graph are defined by

$$
\operatorname{dom} F:=\{x \in X \mid F(x) \neq \emptyset\}
$$

and

$$
\operatorname{gph} F:=\{(x, y) \in X \times Y \mid y \in F(x)\}
$$

respectively. For a mapping $F: X \rightrightarrows X^{*}$ between a Banach space $X$ and its dual $X^{*}$ we define the sequential Painlevé-Kuratowski outer limit by

$$
\operatorname{Limsup}_{x \rightarrow \bar{x}} F(x):=\left\{x^{*} \in X^{*} \mid \exists x_{k} \rightarrow \bar{x} \text { and } x_{k}^{*} \stackrel{w^{*}}{\rightarrow} x^{*} \text { with } x_{k}^{*} \in F\left(x_{k}\right) \forall k\right\} .
$$


Our study is focused on two key notions: metric subregularity and strong metric subregularity. They plays an important role in stability analysis. For more details, one can refer to the references [13-17]. They are defined as follows.

Definition 2.1 A mapping $F: X \rightrightarrows Y$ is said to be metrically subregular at $\bar{x}$ for $\bar{y}$ if $\bar{y} \in F(\bar{x})$ and there is a positive constant $\kappa$ along with a neighborhood $U$ of $\bar{x}$ such that

$$
d\left(x ; F^{-1}(\bar{y})\right) \leq \kappa d(\bar{y} ; F(x)) \text { for all } x \in U
$$

Definition 2.2 A mapping $F: X \rightrightarrows Y$ is said to be strongly (metrically) subregular at $\bar{x}$ for $\bar{y}$ if $\bar{y} \in F(\bar{x})$ and there is a positive constant $\kappa$ along with a neighborhood $U$ of $\bar{x}$ such that

$$
\|x-\bar{x}\| \leq \kappa d(\bar{y} ; F(x)) \quad \text { for all } x \in U
$$

We notice that the strong metric subregularity of $F$ at $\bar{x}$ for $\bar{y}$ is equivalent to the metric subregularity if $\bar{x}$ is an isolated point of $F^{-1}(\bar{y})$.

For an extended-real-valued function $f: X \rightarrow \overline{\mathbb{R}}:=(-\infty ;+\infty]$, we define the domain of $f$ to be

$$
\operatorname{dom} f:=\{x \in X \mid f(x)<+\infty\}
$$

In this paper, unless otherwise stated, we assume that all the extended-real-valued functions are proper, i.e., not identically equal to $+\infty$, and lower semicontinuous (l.s.c.) on $X$.

The fundamental tools for studying general nonsmooth functions are subdifferentials. The following two subdifferential notions are used in this paper.

Definition 2.3 (Subdifferentials of functions) Let $f: X \rightarrow \overline{\mathbb{R}}$ and $\bar{x} \in \operatorname{dom} f$.

- The regular/Fréchet subdifferential of $f$ at $\bar{x}$ is

$$
\hat{\partial} f(\bar{x}):=\left\{x^{*} \in X^{*} \mid \liminf _{x \rightarrow \bar{x}} \frac{f(x)-f(\bar{x})-\left\langle x^{*}, x-\bar{x}\right\rangle}{\|x-\bar{x}\|} \geq 0\right\} .
$$

- The (basic, limiting, Mordukhovich) subdifferential of $f$ at $\bar{x}$ is defined via (2) by

$$
\partial f(\bar{x}):=\operatorname{Limsup}_{x \rightarrow \bar{x}} \hat{\partial} f(x),
$$

where the symbol $x \stackrel{f}{\rightarrow} \bar{x}$ means that $x \rightarrow \bar{x}$ with $f(x) \rightarrow f(\bar{x})$.

When $f$ is convex, $\partial f(x)$ is the usual subgradient set of convex analysis. When $f$ is smooth, $\partial f(x)$ is the singleton $\nabla f(x)$.

Definition 2.4 Given a subset $K \subset X$ and a point $x \in K$. The contingent cone of $K$ at $x$ is defined by

$$
T_{K}(x):=\limsup _{\tau \downarrow 0} \frac{K-x}{\tau}=\left\{v \in X \mid \exists \tau_{k} \rightarrow 0, \exists K \ni x_{k} \rightarrow x \text {, s.t. } \frac{x_{k}-x}{\tau_{k}} \rightarrow v\right\} .
$$


Definition 2.5 (Normal cone) Let there be given a subset $K \subset X$ and a point $\bar{x} \in K$.

- The regular/Fréchet normal cone of $K$ at $\bar{x}$ is defined by

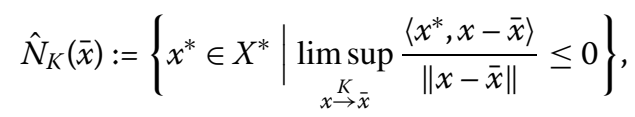

where $\underset{\text { }}{\rightarrow} \stackrel{x}{x}$ signifies the convergence $x \rightarrow \bar{x}$ with $x \in K$.

- The limiting normal cone of $f$ at $\bar{x}$ is defined via (2) by

$$
N_{K}(\bar{x}):=\underset{x^{K} \bar{x}}{\operatorname{Limsup}} \hat{N}_{K}(x) .
$$

When $K$ is convex, $N_{K}(\bar{x})=\hat{N}_{K}(\bar{x})$. Let $C \subset X$ be a cone, we denote by

$$
C^{-}:=\left\{x^{*} \in X^{*} \mid\left\langle x^{*}, x\right\rangle \leq 0 \text { for all } x \in C\right\}
$$

its polar cone. Bonnans and Shapiro [1] pointed out that $\hat{N}_{K}(\bar{x})=\left[T_{K}(\bar{x})\right]^{-}$if $X$ is a finitedimensional space. Applying the last two notions to the subdifferential mapping $\partial f: X \rightrightarrows$ $X^{*}$ for an extended-real-valued function $f$ yields the combined second-order subdifferential of $f$ at $\left(\bar{x}, \bar{x}^{*}\right)$ introduced in [7] by

$$
\breve{\partial}^{2} f\left(\bar{x}, \bar{x}^{*}\right)(u):=\left\{u^{*} \in X^{*} \mid\left(u^{*},-u\right) \in \hat{N}_{\operatorname{gph} \partial f}\left(\bar{x}, \bar{x}^{*}\right)\right\} \quad \text { for all } u \in X^{* *}
$$

and the second-order subdifferential (or generalized Hessian) of $f$ at $\left(\bar{x}, \bar{x}^{*}\right)$ introduced in [18] by

$$
\partial^{2} f\left(\bar{x}, \bar{x}^{*}\right)(u):=\left\{u^{*} \in X^{*} \mid\left(u^{*},-u\right) \in N_{\operatorname{gph} \partial f}\left(\bar{x}, \bar{x}^{*}\right)\right\} \quad \text { for all } u \in X^{* *} .
$$

Note that

$$
\partial^{2} f\left(\bar{x}, \bar{x}^{*}\right)(u)=\left\{\nabla^{2} f(\bar{x})^{*} u\right\} \quad \text { for all } u \in X^{* *}
$$

if $f \in \mathcal{C}^{2}$, where $\nabla^{2} f(\bar{x})^{*}=\nabla^{2} f(\bar{x})$ in the case of Hilbert spaces $X$.

In order to characterize the relationships between the quadratic growth conditions and metric subregularity of the subdifferentials in nonsmooth and nonconvex settings, we need the following two notions, which were introduced in [19] and were further studied in [20] and [21].

Definition 2.6 Let $f: X \rightarrow \overline{\mathbb{R}}$ and $\bar{x} \in \operatorname{dom} f$. We say that $f$ is prox-regular at $\bar{x}$ for $\bar{v} \in \partial f(\bar{x})$ if there exist $\varepsilon>0$ and $r \geq 0$ such that

$$
f\left(x^{\prime}\right) \geq f(x)+\left\langle v, x^{\prime}-x\right\rangle-\frac{r}{2}\left\|x^{\prime}-x\right\|^{2} \quad \text { for all } x^{\prime} \in \mathbb{B}_{\varepsilon}(\bar{x})
$$

whenever $x \in \mathbb{B}_{\varepsilon}(\bar{x}),|f(x)-f(\bar{x})|<\varepsilon$, and $v \in \partial f(x)$ with $\|v-\bar{v}\|<\varepsilon$. If this holds for every $\bar{v} \in \partial f(\bar{x})$, we say that $f$ is prox-regular at $\bar{x}$. 
Definition 2.7 A function $f: X \rightarrow \overline{\mathbb{R}}$ is subdifferentially continuous at $\bar{x} \in \operatorname{dom} f$ for $\bar{v} \in$ $\partial f(\bar{x})$ if for every $\varepsilon>0$ there exists $\delta>0$ such that $|f(x)-f(\bar{x})|<\varepsilon$ whenever $x \in \mathbb{B}_{\delta}(\bar{x})$ and $\|v-\bar{v}\|<\delta$ with $v \in \partial f(x)$. If this occurs for all $\bar{v} \in \partial f(\bar{x})$, we say that $f$ is subdifferentially continuous at $\bar{x}$.

\section{Characterizing quadratic growth properties}

The main focus of this section is relating the quadratic growth properties of a function $f$ to the subregularity of the subdifferential $\partial f$.

In the convex case, Aragón Artacho and Geoffroy [6] gave a condition

$$
f(\lambda x+(1-\lambda) \bar{x}) \leq \lambda f(x)+(1-\lambda) f(\bar{x})-\frac{c}{2} \lambda(1-\lambda)\|x-\bar{x}\|^{2}, \quad \forall x \in U,
$$

where $U$ is a neighborhood of point $\bar{x} \in X, c>0$, and $\lambda \in(0,1)$, which allows the convex subdifferential to be strongly metrically subregular at any $\left(\bar{x}, \bar{x}^{*}\right) \in \operatorname{gph} \partial f$.

It will be showed in the next theorem that a condition similar to (13) guarantees that the upper bound $\frac{1}{\kappa}$ in (1) can be attained.

Theorem 3.1 Let there be given $f: X \rightarrow \overline{\mathbb{R}}$ and $\left(\bar{x}, \bar{x}^{*}\right) \in \operatorname{gph} \partial f$. If the subdifferential $\partial f$ is strongly metrically subregular at $\left(\bar{x}, \bar{x}^{*}\right)$ with modulus $\kappa>0$, then the following two assertions are equivalent:

(i) there are real numbers $r \in\left(0, \kappa^{-1}\right)$ and $\delta>0$ such that

$$
f(x) \geq f(\bar{x})+\left\langle\bar{x}^{*}, x-\bar{x}\right\rangle-\frac{r}{2}\|x-\bar{x}\|^{2} \quad \text { for all } x \in \mathbb{B}_{\delta}(\bar{x}),
$$

(ii) for any real number $\alpha \in\left(0, \kappa^{-1}\right)$, there is a real number $\eta>0$ such that

$$
f(x) \geq f(\bar{x})+\left\langle\bar{x}^{*}, x-\bar{x}\right\rangle+\frac{\alpha}{2}\|x-\bar{x}\|^{2} \quad \text { for all } x \in \mathbb{B}_{\eta}(\bar{x}) .
$$

Furthermore, if there is a neighborhood $U$ of $\bar{x}$ such that for all $x \in U, \lambda \in(0,1)$,

$$
f(\lambda x+(1-\lambda) \bar{x}) \leq \lambda f(x)+(1-\lambda) f(\bar{x})-\frac{1}{2 \kappa} \lambda(1-\lambda)\|x-\bar{x}\|^{2},
$$

then (i) or (ii) can imply the following:

(iii) there is a real number $\theta>0$ such that

$$
f(x) \geq f(\bar{x})+\left\langle\bar{x}^{*}, x-\bar{x}\right\rangle+\frac{1}{2 \kappa}\|x-\bar{x}\|^{2} \quad \text { for all } x \in \mathbb{B}_{\theta}(\bar{x}) .
$$

Proof The proof of the equivalence (i) $\Leftrightarrow$ (ii) appears implicitly in [7], Corollary 3.3. Now, we show the implication (ii) $\Rightarrow$ (iii). Suppose that assertion (iii) is not true, then for any positive integer $k$ such that $\mathbb{B}_{\frac{1}{k}}(\bar{x}) \subset U$, there is an $x_{k} \in \mathbb{B}_{\frac{1}{k}}(\bar{x})$ such that

$$
f\left(x_{k}\right)<f(\bar{x})+\left\langle\bar{x}^{*}, x_{k}-\bar{x}\right\rangle+\frac{1}{2 \kappa}\left\|x_{k}-\bar{x}\right\|^{2} .
$$

One can pick a positive number $\varepsilon_{k}$ such that

$$
f\left(x_{k}\right)<f(\bar{x})+\left\langle\bar{x}^{*}, x_{k}-\bar{x}\right\rangle+\frac{\kappa^{-1}-\varepsilon_{k}}{2}\left\|x_{k}-\bar{x}\right\|^{2} .
$$


Since (ii) holds, for some real number $\kappa^{-1}-\varepsilon_{k}<\alpha_{k}<\kappa^{-1}$, there is a real number $\eta_{k}>0$ such that

$$
f(x) \geq f(\bar{x})+\left\langle\bar{x}^{*}, x-\bar{x}\right\rangle+\frac{\alpha_{k}}{2}\|x-\bar{x}\|^{2} \quad \text { for all } x \in \mathbb{B}_{\eta_{k}}(\bar{x}) .
$$

Next, we show that

$$
f(x)<f(\bar{x})+\left\langle\bar{x}^{*}, x-\bar{x}\right\rangle+\frac{\kappa^{-1}-\varepsilon_{k}}{2}\|x-\bar{x}\|^{2}
$$

for all $x=\lambda x_{k}+(1-\lambda) \bar{x}$ with $\lambda \in(0,1)$. Define

$$
g_{k}(x):=f(x)-\left\langle\bar{x}^{*}, x-\bar{x}\right\rangle-\frac{\kappa^{-1}-\varepsilon_{k}}{2}\|x-\bar{x}\|^{2} .
$$

It follows from (16) and (19) that, for all $\lambda \in(0,1)$,

$$
\begin{aligned}
g_{k}\left(\lambda x_{k}+(1-\lambda) \bar{x}\right)= & f\left(\lambda x_{k}+(1-\lambda) \bar{x}\right)-\lambda\left(\bar{x}^{*}, x_{k}-\bar{x}\right\rangle-\frac{\kappa^{-1}-\varepsilon_{k}}{2} \lambda^{2}\left\|x_{k}-\bar{x}\right\|^{2} \\
\leq & \lambda f\left(x_{k}\right)+(1-\lambda) f(\bar{x})-\frac{1}{2 \kappa} \lambda(1-\lambda)\left\|x_{k}-\bar{x}\right\|^{2} \\
& -\lambda\left(\bar{x}^{*}, x_{k}-\bar{x}\right\rangle-\frac{\kappa^{-1}-\varepsilon_{k}}{2} \lambda^{2}\left\|x_{k}-\bar{x}\right\|^{2} \\
< & \lambda f(\bar{x})+\lambda\left(\bar{x}^{*}, x_{k}-\bar{x}\right\rangle+\frac{\kappa^{-1}-\varepsilon_{k}}{2} \lambda\left\|x_{k}-\bar{x}\right\|^{2}+(1-\lambda) f(\bar{x}) \\
& -\frac{1}{2 \kappa} \lambda(1-\lambda)\left\|x_{k}-\bar{x}\right\|^{2}-\lambda\left(\bar{x}^{*}, x_{k}-\bar{x}\right\rangle-\frac{\kappa^{-1}-\varepsilon_{k}}{2} \lambda^{2}\left\|x_{k}-\bar{x}\right\|^{2} \\
= & f(\bar{x})+\frac{\kappa^{-1}-\varepsilon_{k}}{2} \lambda(1-\lambda)\left\|x_{k}-\bar{x}\right\|^{2}-\frac{1}{2 \kappa} \lambda(1-\lambda)\left\|x_{k}-\bar{x}\right\|^{2} \\
= & f(\bar{x})-\frac{\varepsilon_{k}}{2} \lambda(1-\lambda)\left\|x_{k}-\bar{x}\right\|^{2} \\
< & f(\bar{x}),
\end{aligned}
$$

hence, inequality (21) holds for all $x=\lambda x_{k}+(1-\lambda) \bar{x}$ with $\lambda \in(0,1)$, which contradicts the inequality (20) and thus completes the proof of the theorem.

\section{Remark 3.1}

(i) The condition (16) is a sufficient condition for (17). For example, for $x=\left(x_{1}, x_{2}\right) \in \mathbb{R}^{2}$, consider the function

$$
f(x):= \begin{cases}+\infty, & x_{1}=x_{2} \neq 0 \\ \frac{1}{2}\|x\|^{2}, & \text { otherwise }\end{cases}
$$

Obviously, $f$ is not convex, and the subdifferential $\partial f$ is strongly metrically subregular at $\left(\bar{x}, \bar{x}^{*}\right)=(\mathbf{0}, \mathbf{0}) \in \operatorname{gph} \partial f$ with modulus $\kappa=1$. Furthermore, we observe that, for any neighborhood $U$ of $\bar{x}=(0,0)$ and $\lambda \in(0,1)$, the condition (16) holds. On the other hand, we see that (17) holds at $\left(\bar{x}, \bar{x}^{*}\right)=(\mathbf{0}, \mathbf{0})$. 
(ii) The condition (16) is only a sufficient condition for (17). Consider the real function $f(x)=|x|$. We observe that the subgradient mapping $\partial f$ is a strongly metrically subregular at $\bar{x}=0$ for $\bar{x}^{*}=0$ with any $\kappa>0$, and inequality (17) is satisfied at $(0,0)$. However, the inequality (16) does not hold.

(iii) We observe that the bound $\alpha=\kappa^{-1}$ in (15) is the sharpest possible bound. To illustrate this, consider the real function $f(x)=\frac{1}{2} x^{2}$. We observe that the subgradient mapping $\partial f$ is strongly metrically subregular at $\bar{x}=0$ for $\bar{x}^{*}=0$ with modulus $\kappa=1$, and inequality (15) is satisfied at $(0,0)$ for $\alpha=\kappa^{-1}$. However, inequality $(15)$ is false at $(0,0)$ for any $\alpha>\kappa^{-1}$.

(iv) Notice that condition (16) is weaker than the strong convexity of $f$ in $U$, because the strong convexity entails the condition that there exists a constant $c$ such that, for all $x_{1}, x_{2} \in U$ and $\lambda \in(0,1)$,

$$
f\left(\lambda x_{1}+(1-\lambda) x_{2}\right) \leq \lambda f\left(x_{1}\right)+(1-\lambda) f\left(x_{2}\right)-c \lambda(1-\lambda)\left\|x_{1}-x_{2}\right\|^{2} .
$$

In a nonconvex setting, Drusvyatskiy et al. [7] showed that the quadratic growth property (15) holds if the subdifferential $\partial f$ is metrically subregular at $\left(\bar{x}, \bar{x}^{*}\right)$ with $\kappa^{-1}>0$ and the inequality (14) holds. Furthermore, we have an additional uniformity condition: there exists $\beta \in[0, \alpha)$ such that

$$
f(\bar{x}) \geq f(x)+\left\langle x^{*}, \bar{x}-x\right\rangle-\frac{\beta}{2}\|x-\bar{x}\|^{2}, \quad \forall\left(x, x^{*}\right) \in \operatorname{gph} \partial f \cap \mathbb{B}_{\eta}\left(\bar{x}, \bar{x}^{*}\right),
$$

which together with (15) implies that the subdifferential $\partial f$ is strongly metrically subregular. We notice that the quadratic growth property of a proper lower semicontinuous function $f$ can also hold if we substitute the strong metric subregularity of $\partial f$ with a condition similar to (13). More precisely, we have the following theorems.

Theorem 3.2 Let there be given $f: X \rightarrow \overline{\mathbb{R}},\left(\bar{x}, \bar{x}^{*}\right) \in \operatorname{gph} \partial f$, and $\kappa>0$. If there are real numbers $r \in\left(0, \kappa^{-1}\right)$ and $\theta>0$ such that

$$
f(x) \geq f(\bar{x})+\left\langle\bar{x}^{*}, x-\bar{x}\right\rangle-\frac{r}{2} d^{2}\left(x ;(\partial f)^{-1}\left(\bar{x}^{*}\right)\right) \quad \text { for all } x \in \mathbb{B}_{\theta}(\bar{x}),
$$

and for any real number $\alpha \in\left(0, \kappa^{-1}\right)$, there is a positive real number $\mu$ such that, for all $x \in \mathbb{B}_{\mu}(\bar{x})$ and $\lambda \in(0,1)$,

$$
f((1-\lambda) x+\lambda \bar{x}) \leq(1-\lambda) f(x)+\lambda f(\bar{x})-\frac{\alpha}{2} \lambda(1-\lambda) d^{2}\left(x ;(\partial f)^{-1}\left(\bar{x}^{*}\right)\right),
$$

then there is a real number $\eta>0$ such that

$$
f(x) \geq f(\bar{x})+\left\langle\bar{x}^{*}, x-\bar{x}\right\rangle+\frac{\alpha}{2} d^{2}\left(x ;(\partial f)^{-1}\left(\bar{x}^{*}\right)\right) \quad \text { for all } x \in \mathbb{B}_{\eta}(\bar{x}) .
$$

Furthermore, if there are a real number $\beta \in(0, \alpha)$ and a positive real number $\delta$ such that

$$
\begin{gathered}
f(u) \geq f(x)+\left\langle x^{*}, u-x\right\rangle-\frac{\beta}{2} d^{2}\left(x ;(\partial f)^{-1}\left(\bar{x}^{*}\right)\right), \\
\forall\left(u, \bar{x}^{*}\right),\left(x, x^{*}\right) \in \operatorname{gph} \partial f \cap \mathbb{B}_{\delta}\left(\bar{x}, \bar{x}^{*}\right),
\end{gathered}
$$

then the subdifferential $\partial f$ is metrically subregular at $\left(\bar{x}, \bar{x}^{*}\right)$. 
Proof Let $\vartheta=\min \{\theta, \mu\}$. It follows from (26) that, for all $x \in \mathbb{B}_{\vartheta}(\bar{x})$ and $\lambda \in(0,1)$,

$$
(1-\lambda) f(x) \geq f((1-\lambda) x+\lambda \bar{x})-\lambda f(\bar{x})+\frac{\alpha}{2} \lambda(1-\lambda) d^{2}\left(x ;(\partial f)^{-1}\left(\bar{x}^{*}\right)\right),
$$

i.e.,

$$
f(x) \geq \frac{1}{1-\lambda} f((1-\lambda) x+\lambda \bar{x})-\frac{\lambda}{1-\lambda} f(\bar{x})+\frac{\alpha}{2} \lambda d^{2}\left(x ;(\partial f)^{-1}\left(\bar{x}^{*}\right)\right) .
$$

Moreover, by (25),

$$
f((1-\lambda) x+\lambda \bar{x}) \geq f(\bar{x})+(1-\lambda)\left\langle\bar{x}^{*}, x-\bar{x}\right\rangle-\frac{r}{2}(1-\lambda)^{2}\|x-\bar{x}\|^{2},
$$

which together with the inequality (29) gives

$$
f(x) \geq f(\bar{x})+\left\langle\bar{x}^{*}, x-\bar{x}\right\rangle+\frac{\alpha}{2} \lambda d^{2}\left(x ;(\partial f)^{-1}\left(\bar{x}^{*}\right)\right)-\frac{r}{2}(1-\lambda)\|x-\bar{x}\|^{2} .
$$

Letting $\lambda \uparrow 1$ in this inequality, we obtain (27). By applying Theorem 3.1 of [7], it follows from (27) and (28) that the subdifferential $\partial f$ is metrically subregular at $\left(\bar{x}, \bar{x}^{*}\right)$.

Furthermore, it is easy to observe the following consequence of Theorem 3.2 concerning the strong metric subregularity of the subdifferential.

Corollary 3.1 Let there be given $f: X \rightarrow \overline{\mathbb{R}},\left(\bar{x}, \bar{x}^{*}\right) \in \operatorname{gph} \partial f$, and $\kappa>0$. If there are real numbers $r \in\left(0, \kappa^{-1}\right)$ and $\theta>0$ such that

$$
f(x) \geq f(\bar{x})+\left\langle\bar{x}^{*}, x-\bar{x}\right\rangle-\frac{r}{2}\|x-\bar{x}\|^{2} \quad \text { for all } x \in \mathbb{B}_{\theta}(\bar{x})
$$

and for any real number $\alpha \in\left(0, \kappa^{-1}\right)$, there is a positive real number $\mu$ such that for all $x \in \mathbb{B}_{\mu}(\bar{x})$ and $\lambda \in(0,1)$,

$$
f((1-\lambda) x+\lambda \bar{x}) \leq(1-\lambda) f(x)+\lambda f(\bar{x})-\frac{\alpha}{2} \lambda(1-\lambda)\|x-\bar{x}\|^{2},
$$

then there is a real number $\eta>0$ such that

$$
f(x) \geq f(\bar{x})+\left\langle\bar{x}^{*}, x-\bar{x}\right\rangle+\frac{\alpha}{2}\|x-\bar{x}\|^{2} \quad \text { for all } x \in \mathbb{B}_{\eta}(\bar{x}) .
$$

Furthermore, if there are a real number $\beta \in(0, \alpha)$ and a positive real number $\delta$ such that

$$
f(\bar{x}) \geq f(x)+\left\langle x^{*}, \bar{x}-x\right\rangle-\frac{\beta}{2}\|x-\bar{x}\|^{2}, \quad \forall\left(x, x^{*}\right) \in \operatorname{gph} \partial f \cap \mathbb{B}_{\delta}\left(\bar{x}, \bar{x}^{*}\right),
$$

then we see that the subdifferential $\partial$ is strongly metrically subregular at $\left(\bar{x}, \bar{x}^{*}\right)$.

The conditions (25) and (28) can be weakened, if we strengthen slightly the condition (26). More specifically, we have the following result. 
Theorem 3.3 Let there be given $f: X \rightarrow \overline{\mathbb{R}},\left(\bar{x}, \bar{x}^{*}\right) \in \operatorname{gph} \partial f$, and $\kappa>0$. If there are real numbers $r \in\left(0, \kappa^{-1}\right)$ and $\theta>0$ such that

$$
f(x) \geq f(\bar{x})+\left\langle\bar{x}^{*}, x-\bar{x}\right\rangle-\frac{r}{2}\|x-\bar{x}\|^{2} \quad \text { for all } x \in \mathbb{B}_{\theta}(\bar{x}),
$$

and for any real number $\alpha \in\left(0, \kappa^{-1}\right)$, there is a positive real number $\mu$ such that for all $x \in \mathbb{B}_{\mu}(\bar{x})$ and $\lambda \in(0,1)$,

$$
f((1-\lambda) x+\lambda \bar{x}) \leq(1-\lambda) f(x)+\lambda f(\bar{x})-\frac{\alpha}{2} \lambda(1-\lambda) d\left(x ;(\partial f)^{-1}\left(\bar{x}^{*}\right)\right)\|x-\bar{x}\|,
$$

then there is a real number $\eta>0$ such that

$$
f(x) \geq f(\bar{x})+\left\langle\bar{x}^{*}, x-\bar{x}\right\rangle+\frac{\alpha}{2} d^{2}\left(x ;(\partial f)^{-1}\left(\bar{x}^{*}\right)\right) \quad \text { for all } x \in \mathbb{B}_{\eta}(\bar{x}) .
$$

Furthermore, if there are a real number $\beta \in(0, \alpha)$ and a positive real number $\delta$ such that

$$
f(\bar{x}) \geq f(x)+\left\langle x^{*}, \bar{x}-x\right\rangle-\frac{\beta}{2} d\left(x ;(\partial f)^{-1}\left(\bar{x}^{*}\right)\right)\|x-\bar{x}\|, \quad \forall\left(x, x^{*}\right) \in \operatorname{gph} \partial f \cap \mathbb{B}_{\delta}\left(\bar{x}, \bar{x}^{*}\right),
$$

then the subdifferential $\partial f$ is metrically subregular at $\left(\bar{x}, \bar{x}^{*}\right)$.

Proof Let $\vartheta=\min \{\theta, \mu\}$. For all $x \in \mathbb{B}_{\vartheta}(\bar{x})$ and $\lambda \in(0,1)$, the inequality (36) gives

$$
f((1-\lambda) x+\lambda \bar{x}) \geq f(\bar{x})+(1-\lambda)\left(\bar{x}^{*}, x-\bar{x}\right\rangle-\frac{r}{2}(1-\lambda)^{2}\|x-\bar{x}\|^{2} .
$$

It follows from (37) that, for all $x \in \mathbb{B}_{\vartheta}(\bar{x})$ and $\lambda \in(0,1)$,

$$
f(x) \geq \frac{1}{1-\lambda} f((1-\lambda) x+\lambda \bar{x})-\frac{\lambda}{1-\lambda} f(\bar{x})+\frac{\alpha}{2} \lambda d\left(x ;(\partial f)^{-1}\left(\bar{x}^{*}\right)\right)\|x-\bar{x}\|,
$$

which together with the inequality $(40)$ gives

$$
f(x) \geq f(\bar{x})+\left\langle\bar{x}^{*}, x-\bar{x}\right\rangle+\frac{\alpha}{2} \lambda d\left(x ;(\partial f)^{-1}\left(\bar{x}^{*}\right)\right)\|x-\bar{x}\|-\frac{r}{2}(1-\lambda)\|x-\bar{x}\|^{2} .
$$

Letting $\lambda \uparrow 1$ in this inequality, we have

$$
f(x) \geq f(\bar{x})+\left\langle\bar{x}^{*}, x-\bar{x}\right\rangle+\frac{\alpha}{2} d\left(x ;(\partial f)^{-1}\left(\bar{x}^{*}\right)\right)\|x-\bar{x}\|, \quad \forall x \in \mathbb{B}_{\vartheta}(\bar{x}),
$$

which implies (38).

Furthermore, letting $\varepsilon=\min \{\vartheta, \delta\}$, it follows from (39) and (43) that, for all $\left(x, x^{*}\right) \in$ $\operatorname{gph} \partial f \cap \mathbb{B}_{\varepsilon}\left(\bar{x}, \bar{x}^{*}\right)$,

$$
\left\langle x^{*}-\bar{x}^{*}, x-\bar{x}\right\rangle \geq \frac{\alpha-\beta}{2} d\left(x ;(\partial f)^{-1}\left(\bar{x}^{*}\right)\right)\|x-\bar{x}\|,
$$

which implies

$$
d\left(x ;(\partial f)^{-1}\left(\bar{x}^{*}\right)\right) \leq \frac{2}{\alpha-\beta}\left\|x^{*}-\bar{x}^{*}\right\|, \quad \forall x \in \mathbb{B}_{\varepsilon}(\bar{x}), \forall x^{*} \in \partial f(x) .
$$

Thus the subdifferential $\partial f$ is metrically subregular at $\left(\bar{x}, \bar{x}^{*}\right)$. 


\section{Second-order characterizations of quadratic growth properties}

In this section, we characterize the quadratic growth properties by the second-order subdifferential (or generalized Hessian) defined in (10). To motivate the subsequent discussion, we first review the standard second-order optimality condition and restate it in terms of the gradient itself. The proof of the following proposition is similar to [22], Proposition 5.1.

Proposition 4.1 Consider a $\mathcal{C}^{2}$-smooth function $f$ on $\mathbb{R}^{n}$ and let $\bar{x}$ be a local minimizer off. Then the following conditions are equivalent:

(i) the gradient mapping $\nabla f$ is strongly metrically subregular at $(\bar{x}, 0)$,

(ii) the Hessian matrix $\nabla^{2} f(\bar{x})$ is positive definite.

Proof Since $f$ is $\mathcal{C}^{2}$-smooth, we have

$$
\nabla f(x)=\nabla^{2} f(\bar{x})(x-\bar{x})+o(\|x-\bar{x}\|)
$$

and hence

$$
\|\nabla f(x)+r(x-\bar{x})\|=\left\|\left(\nabla^{2} f(\bar{x})+r I\right)(x-\bar{x})\right\|+o(\|x-\bar{x}\|) \quad \text { for all } r \geq 0,
$$

where the symbol $I$ denote the identity mapping. If the Hessian matrix $\nabla^{2} f(\bar{x})$ is positive definite, there are a real number $\varepsilon>0$ and a positive real number $\lambda$ which is slightly smaller than the minimal eigenvalue of $\nabla^{2} f(\bar{x})$ such that

$$
\|\nabla f(x)+r(x-\bar{x})\| \geq(\lambda+r)\|x-\bar{x}\| \quad \text { for all } x \in \mathbb{B}_{\varepsilon}(\bar{x}) .
$$

It follows from the last inequality that

$$
\lambda\|x-\bar{x}\| \leq\|\nabla f(x)\| \quad \text { for all } x \in \mathbb{B}_{\varepsilon}(\bar{x}),
$$

which verifies the gradient mapping $\nabla f$ is strongly metrically subregular at $(\bar{x}, 0)$ with modulus $\lambda^{-1}$. Hence the implication (ii) $\Rightarrow$ (i) holds.

To show the implication (i) $\Rightarrow$ (ii), suppose the gradient mapping $\nabla f$ is strongly metrically subregular at $(\bar{x}, 0)$ with $\kappa>0$, then there is a real number $\delta>0$ such that

$$
\|x-\bar{x}\| \leq \kappa\|\nabla f(x)\| \quad \text { for all } x \in \mathbb{B}_{\delta}(\bar{x})
$$

which implies that one can pick a real number $r \in\left[0, \frac{1}{2 \kappa}\right)$ such that

$$
\|\nabla f(x)+r(x-\bar{x})\| \geq\left(\kappa^{-1}-r\right)\|x-\bar{x}\| \quad \text { for all } x \in \mathbb{B}_{\delta}(\bar{x}) .
$$

Hence there exists a positive real number $\mu$ such that

$$
\|\nabla f(x)+r(x-\bar{x})\| \geq(\mu+r)\|x-\bar{x}\| \quad \text { for all } x \in \mathbb{B}_{\delta}(\bar{x}) .
$$

If the Hessian matrix $\nabla^{2} f(\bar{x})$ is not positive definite, then there is an eigenvalue $\bar{\lambda}=0$ and a corresponding eigenvector $v \in \mathbb{R}^{n}$ with $\|v\|=1$. Let $x_{t}=\bar{x}+t v$, then $x_{t} \in \mathbb{B}_{\delta}(\bar{x})$ when $t$ is 
small enough. It follows from (44) that

$$
\begin{aligned}
(\mu+r) t & \leq\left\|\nabla f\left(x_{t}\right)+r\left(x_{t}-\bar{x}\right)\right\|=\left\|\left(\nabla^{2} f(\bar{x})+r I\right)\left(x_{t}-\bar{x}\right)\right\|+o\left(\left\|x_{t}-\bar{x}\right\|\right) \\
& \leq r t+o(t)
\end{aligned}
$$

which is a contradiction and thus completes the proof.

In a nonsmooth setting, it is natural to use a second-order subdifferential construction. The next theorem establishes a relationship among the strong metric subregularity, the quadratic growth condition, and the positive-definiteness property of the combined second-order subdifferential for a prox-regular and subdifferentially continuous function. Its proof technique follows [6], Corollary 3.7.

Definition 4.1 Let there be given $f: \mathbb{R}^{n} \rightarrow \overline{\mathbb{R}}$ and $\left(\bar{x}, \bar{x}^{*}\right) \in \operatorname{gph} \partial f$. We say that $f$ is contingent-normal at $\bar{x}$ for $\bar{x}^{*}$ if the following property holds: for a vector $u \in \mathbb{R}^{n}$, if there exists a vector $w^{*}$ such that $\left(u, w^{*}\right) \in T_{\operatorname{gph} \partial f}\left(\bar{x}, \bar{x}^{*}\right)$, then there is a vector $u^{*}$ such that $\left(u^{*},-u\right) \in \hat{N}_{\mathrm{gph} \partial f}\left(\bar{x}, \bar{x}^{*}\right)$.

Definition 4.2 Let there be given $f: \mathbb{R}^{n} \rightarrow \overline{\mathbb{R}}$ and $\left(\bar{x}, \bar{x}^{*}\right) \in \operatorname{gph} \partial f$. We say that $f$ is normalcontingent at $\bar{x}$ for $\bar{x}^{*}$ if the following property holds: for a vector $u \in \mathbb{R}^{n}$, if there exists a vector $u^{*}$ such that $\left(u^{*},-u\right) \in \hat{N}_{\text {gph } \partial f}\left(\bar{x}, \bar{x}^{*}\right)$, then there is a vector $w^{*}$ such that $\left(-u,-w^{*}\right) \in$ $T_{\mathrm{gph} \partial f}\left(\bar{x}, \bar{x}^{*}\right)$.

Remark 4.1 We observe that the assumptions in Definition 4.1 and Definition 4.2 can be satisfied automatically by many functions. For instance, if gph $\partial f$ has the form of the following set:

$$
\bigcup\left\{(x, y) \in \mathbb{R}^{n} \times \mathbb{R}_{+}^{n} \mid\left\langle\left(a_{i}, b_{i}\right),(x, y)\right\rangle=0,\left(a_{i}, b_{i}\right) \in\left(\mathbb{R}^{n} \backslash\{0\}\right) \times \mathbb{R}_{--}^{n}, i=1,2, \ldots, p\right\},
$$

then the function $f$ is contingent-normal and normal-contingent at any point $\left(\bar{x}, \bar{x}^{*}\right) \in$ gph $\partial f$. For example, consider the real-valued function

$$
f(x)= \begin{cases}\frac{1}{2} x^{2}, & x \geq 0 \\ -\frac{1}{2} x^{2}, & x<0 .\end{cases}
$$

Then $\nabla f(x)=|x|$ and

$$
\begin{aligned}
\operatorname{gph} \nabla f= & \left\{(x, y) \in \mathbb{R} \times \mathbb{R}_{+} \mid\langle(1,-1),(x, y)\rangle=0\right\} \\
& \cup\left\{(x, y) \in \mathbb{R} \times \mathbb{R}_{+} \mid\langle(-1,-1),(x, y)\rangle=0\right\},
\end{aligned}
$$

which is a form of $(45)$. Take $\left(\bar{x}, \bar{x}^{*}\right)=(0,0)$, we have

$$
T_{\mathrm{gph} \nabla f}\left(\bar{x}, \bar{x}^{*}\right)=\operatorname{gph} \nabla f \quad \text { and } \quad \hat{N}_{\mathrm{gph} \nabla f}\left(\bar{x}, \bar{x}^{*}\right)=\left[T_{\mathrm{gph} \nabla f}\left(\bar{x}, \bar{x}^{*}\right)\right]^{-}
$$

It is easy to verify that this function $f$ is contingent-normal and normal-contingent at $(0,0)$. 
Theorem 4.1 Let there be given $f: \mathbb{R}^{n} \rightarrow \overline{\mathbb{R}}$ and $\left(\bar{x}, \bar{x}^{*}\right) \in \operatorname{gph} \partial f$. Assume $f$ is prox-regular at $\bar{x}$ for $\bar{x}^{*}$ with $r>0$ and subdifferentially continuous at $\bar{x}$ for $\bar{x}^{*}$. Consider the following three statements:

(i) the subdifferential $\partial f$ is strongly metrically subregular at $\left(\bar{x}, \bar{x}^{*}\right)$ with modulus $\kappa>0$,

(ii) there exist a neighborhood $U$ of $\bar{x}$ and a positive constant $\alpha>r$ such that

$$
f(x) \geq f(\bar{x})+\left\langle\bar{x}^{*}, x-\bar{x}\right\rangle+\frac{\alpha}{2}\|x-\bar{x}\|^{2} \quad \text { for all } x \in U,
$$

(iii) there is a constant $c>0$ such that the combined second-order subdifferential $\breve{\partial}^{2} f\left(\bar{x}, \bar{x}^{*}\right)$ is positive-definite in the sense that

$$
\left\langle u^{*}, u\right\rangle \geq c\|u\|^{2} \quad \text { for all } u \in X \text { and } u^{*} \in \breve{\partial}^{2} f\left(\bar{x}, \bar{x}^{*}\right)(u) \text {. }
$$

Then implication (i) $\Rightarrow$ (ii) holds when $\kappa^{-1}>r$. Implication (ii) $\Rightarrow$ (iii) holds with $c=\frac{\alpha-r}{2}$ iff is normal-contingent at $\bar{x}$ for $\bar{x}^{*}$. Furthermore, (iii) $\Rightarrow$ (i) holds with $\kappa>\frac{1}{c}$ iff is contingentnormal at $\bar{x}$ for $\bar{x}^{*}$.

Proof The implication (i) $\Rightarrow$ (ii) has been established in [23], Theorem 12. Next, we show the implication (iii) $\Rightarrow$ (i). Our immediate goal now is to show that for any $\kappa>1 / c$, there are some neighborhoods $U$ of $\bar{x}$ and $V$ of $\bar{x}^{*}$ such that

$$
\|x-\bar{x}\| \leq \kappa\left\|x^{*}-\bar{x}^{*}\right\|, \quad \text { whenever }\left(x, x^{*}\right) \in(\operatorname{gph} \partial f) \cap(U \times V) .
$$

If it is not the case, then for all $n \in \mathbb{N}$ there is $\left(x_{n}, x_{n}^{*}\right) \in \operatorname{gph} \partial f$ with $\left\|x_{n}-\bar{x}\right\| \leq \frac{1}{n}$, $\left\|x_{n}^{*}-\bar{x}^{*}\right\| \leq \frac{1}{n}$ and such that $\left\|x_{n}-\bar{x}\right\|>\kappa\left\|x_{n}^{*}-\bar{x}^{*}\right\|$. This implies $x_{n} \neq \bar{x}$ for all $n$, hence the bounded sequence $\left(\frac{x_{n}-\bar{x}}{\left\|x_{n}-\bar{x}\right\|}, \frac{x_{n}^{*}-\bar{x}^{*}}{\left\|x_{n}-\bar{x}\right\|}\right)$ must have a convergent subsequence. Without loss of generality, we may assume that the sequence $\left(\frac{x_{n}-\bar{x}}{\left\|x_{n}-\bar{x}\right\|}, \frac{x_{n}^{*}-\bar{x}^{*}}{\left\|x_{n}-\bar{x}\right\|}\right)$ is convergent, hence there are some points $u$ and $w^{*}$ with

$$
\left(\frac{x_{n}-\bar{x}}{\left\|x_{n}-\bar{x}\right\|}, \frac{x_{n}^{*}-\bar{x}^{*}}{\left\|x_{n}-\bar{x}\right\|}\right) \rightarrow\left(u, w^{*}\right) \in T_{\operatorname{gph} \partial f}\left(\bar{x}, \bar{x}^{*}\right) .
$$

Since $f$ is contingent-normal at $\bar{x}$ for $\bar{x}^{*}$, there is a vector $u^{*}$ such that $\left(u^{*},-u\right) \in$ $\hat{N}_{\text {gph } \partial f}\left(\bar{x}, \bar{x}^{*}\right)=\left[T_{\text {gph } \partial f}\left(\bar{x}, \bar{x}^{*}\right)\right]^{-}$, i.e., $u^{*} \in \breve{\partial}^{2} f\left(\bar{x}, \bar{x}^{*}\right)(u)$, thus we have

$$
\left\langle\left(u^{*},-u\right),\left(u, w^{*}\right)\right\rangle \leq 0, \quad \text { i.e., } \quad\left\langle u^{*}, u\right\rangle \leq\left\langle w^{*}, u\right\rangle,
$$

this together with (47) gives $\left\langle w^{*}, u\right\rangle \geq c\|u\|^{2}$, thus we have

$$
1=\|u\| \geq \kappa\left\|w^{*}\right\| \geq c \kappa\|u\|>1 .
$$

This is a clear contradiction, which shows condition (48) holds. Thus, the subdifferential $\partial f$ is strongly metrically subregular at $\left(\bar{x}, \bar{x}^{*}\right)$ with any constant $\kappa>1 / c$.

Finally, we prove the implication (ii) $\Rightarrow$ (iii). Since (ii) holds, there exist a neighborhood $U$ of $\bar{x}$ and a positive constant $\alpha$ such that (46) holds. Let $u \in \mathbb{R}^{n}$ and consider 
$u^{*} \in \breve{\partial}^{2} f\left(\bar{x}, \bar{x}^{*}\right)(u)$, i.e., $\left(u^{*},-u\right) \in \hat{N}_{\mathrm{gph} \partial f}\left(\bar{x}, \bar{x}^{*}\right)$. Since $f$ is normal-contingent at $\bar{x}$ for $\bar{x}^{*}$, there is a vector $w^{*}$ such that $\left(-u,-w^{*}\right) \in T_{\operatorname{gph} \partial f}\left(\bar{x}, \bar{x}^{*}\right)$, thus we have

$$
\left\langle\left(u^{*},-u\right),\left(-u,-w^{*}\right)\right\rangle \leq 0, \quad \text { i.e., } \quad\left\langle u^{*}, u\right\rangle \geq\left\langle w^{*}, u\right\rangle,
$$

and there are $\left(x_{n}, x_{n}^{*}\right) \in \operatorname{gph} \partial f$ and $\tau_{n} \downarrow 0$ with $\left(x_{n}, x_{n}^{*}\right) \rightarrow\left(\bar{x}, \bar{x}^{*}\right)$ and

$$
\left[\left(x_{n}, x_{n}^{*}\right)-\left(\bar{x}, \bar{x}^{*}\right)\right] / \tau_{n} \rightarrow\left(-u,-w^{*}\right) .
$$

Let $x \in U$ and consider $x^{*} \in \partial f(x)$, we have

$$
\left\langle x^{*}, x-\bar{x}\right\rangle \geq f(x)-f(\bar{x})-\frac{r}{2}\|x-\bar{x}\|^{2},
$$

this together with (46) gives

$$
\left\langle x^{*}-\bar{x}^{*}, x-\bar{x}\right\rangle \geq \frac{\alpha-r}{2}\|x-\bar{x}\|^{2} \quad \text { for all } x \in U, x^{*} \in \partial f(x) .
$$

Since $x_{n}^{*} \in \partial f\left(x_{n}\right)$ and $x_{n} \in U$ when $n$ is large enough, one has

$$
\left\langle x_{n}^{*}-\bar{x}^{*}, x_{n}-\bar{x}\right\rangle \geq \frac{\alpha-r}{2}\left\|x_{n}-\bar{x}\right\|^{2}
$$

when $n$ is large enough. Hence,

$$
\left\langle\frac{x_{n}^{*}-\bar{x}^{*}}{\tau_{n}}, \frac{x_{n}-\bar{x}}{\tau_{n}}\right\rangle \geq \frac{\alpha-r}{2}\left\|\frac{x_{n}-\bar{x}}{\tau_{n}}\right\|^{2}, \quad \text { eventually. }
$$

Letting $n \rightarrow+\infty$ we have $\left\langle w^{*}, u\right\rangle \geq \frac{\alpha-r}{2}\|u\|^{2}$, this together with (50) yields $\left\langle u^{*}, u\right\rangle \geq$ $\frac{\alpha-r}{2}\|u\|^{2}$, which justifies that the combined second-order subdifferential $\breve{\partial}^{2} f\left(\bar{x}, \bar{x}^{*}\right)$ is positive-definite and thus completes the proof of the theorem.

In the following theorem, we establish an equivalence among the strong metric subregularity, the quadratic growth condition, the positive-definiteness property of the secondorder subdifferential/generalized Hessian, the strong metric regularity, and tilt stability for a proper l.s.c. function acting in $\mathbb{R}^{n}$.

Theorem 4.2 Consider a proper l.s.c. function $f: \mathbb{R}^{n} \rightarrow \overline{\mathbb{R}}$ and a point $(\bar{x}, 0) \in \operatorname{gph} \partial f$. If $f$ is normal-contingent at $\bar{x}$ for $\bar{x}^{*}=0$ and gph $\partial f$ is convex, then the following statements are equivalent:

(i) the subgradient mapping $\partial f$ is strongly metrically subregular at $(\bar{x}, 0)$ with $\kappa>0$,

(ii) there is a constant $c>0$ such that the second-order subdifferential $\partial^{2} f(\bar{x}, 0)$ is positive-definite in the sense that

$$
\left\langle u^{*}, u\right\rangle \geq c\|u\|^{2} \quad \text { for all } u \in X \text { and } u^{*} \in \partial^{2} f(\bar{x}, 0)(u),
$$

(iii) there exist neighborhoods $U$ of $\bar{x}$ and $V$ of 0 such that for any $u^{*} \in V$ there is a point $u \in(\partial f)^{-1}\left(u^{*}\right) \cap U$ satisfying the inequality

$$
f(x) \geq f(u)+\left\langle u^{*}, x-u\right\rangle+\frac{1}{2 \kappa}\|x-u\|^{2} \quad \text { for all } x \in U,
$$


(iv) the subgradient mapping $\partial f$ is strongly metrically regular around $(\bar{x}, 0)$ with $\kappa>0$,

(v) the second-order subdifferential $\partial^{2} f(\bar{x}, 0)$ is positive-definite in the sense that

$$
\left\langle u^{*}, u\right\rangle>0 \quad \text { for all } u \in X \text { and } u^{*} \in \partial^{2} f(\bar{x}, 0)(u), u \neq 0,
$$

(vi) the point $\bar{x}$ is a tilt-stable local minimizer of the function $f$.

Proof Since gph $\partial f$ is convex, we have $\hat{N}_{\operatorname{gph} \partial f}\left(\bar{x}, \bar{x}^{*}\right)=N_{\operatorname{gph} \partial f}\left(\bar{x}, \bar{x}^{*}\right)$. Thereby, $\breve{\partial}^{2} f\left(\bar{x}, \bar{x}^{*}\right)(u)=$ $\partial^{2} f\left(\bar{x}, \bar{x}^{*}\right)(u)$, which together with Theorem 4.1 shows that the implications (i) $\Rightarrow$ (ii) holds. The equivalence among (iii), (iv), (v), and (vi) has been established by Drusvyatskiy et al. [7]. The implications (ii) $\Rightarrow$ (v) and (iv) $\Rightarrow$ (i) hold trivially.

\section{Remark 4.2}

(i) Under the assumptions of Theorem 4.2, we see that the constant $c$ in the quadratic growth condition (1) can attain to $\frac{1}{\kappa}$, and the strong metric subregularity is equivalent to the strong metric regularity.

(ii) The assumption that gph $\partial f$ is convex is essential in the conclusion of Theorem 4.2. If this assumption fails, the equivalence between the strong metric subregularity and the strong metric regularity may be a failure. To illustrate it, take the real-valued function $f(x)=|x|$, we observe that $\partial f$ is strongly metrically subregular at $(0,0)$ with any $\kappa>0$ and $f$ is normal-contingent at $\bar{x}=0$ for $\bar{x}^{*}=0$ as well as gph $\partial f$ is not convex. On the other hand, $\partial f$ is not strongly metrically regular at $(0,0)$.

\section{Competing interests}

The authors declare that they have no competing interests.

\section{Authors' contributions}

All authors contributed equally to the writing of this paper. All authors read and approved the final manuscript.

\section{Author details}

${ }^{1}$ Department of Mathematics, Harbin Institute of Technology, Harbin, 150001, China. ${ }^{2}$ College of Computer Science and Information Engineering, Harbin Normal University, Harbin, 150025, China. ${ }^{3}$ School of Mathematical Sciences, Harbin Normal University, Harbin, 150025, China.

\section{Acknowledgements}

This work was supported by the National Natural Sciences Grant (No. 11371116).

Received: 11 October 2015 Accepted: 15 January 2016 Published online: 27 January 2016

\section{References}

1. Bonnans, JF, Shapiro, A: Perturbation Analysis of Optimization Problems. Springer, New York (2000)

2. Nocedal, J, Wright, SJ: Numerical Optimization. Springer Series in Operations Research and Financial Engineering. Springer, New York (2006)

3. Ruszczyński, A: Nonlinear Optimization. Princeton University Press, Princeton (2006)

4. Aragón Artacho, FJ, Geoffroy, MH: Characterization of metric regularity of subdifferentials. J. Convex Anal. 15(2), 365-380 (2008)

5. Mordukhovich, BS, Nghia, TTA: Second-order variational analysis and characterizations of tilt-stable optimal solutions in finite and infinite dimensions. Nonlinear Anal. 86, 159-180 (2013)

6. Aragón Artacho, FJ, Geoffroy, MH: Metric subregularity of the convex subdifferential in Banach spaces. J. Nonlinear Convex Anal. 15(1), 35-47 (2014)

7. Drusvyatskiy, D, Mordukhovich, BS, Nghia, TTA: Second-order growth, tilt stability and metric regularity of the subdifferential. J. Convex Anal. 21(4), 301-324 (2014)

8. Poliquin, RA, Rockafellar, RT: Tilt stability of a local minimum. SIAM J. Optim. 8(2), 287-299 (1998)

9. Borwein, JM, Zhu, QJ: Techniques of Variational Analysis. Springer, New York (2005)

10. Clarke, FH, Ledyaev, Y, Stern, RI, Wolenski, PR: Nonsmooth Analysis and Control Theory. Springer, New York (1998)

11. Penot, JP: Calculus Without Derivatives. Graduate Texts in Mathematics. Springer, New York (2013)

12. Rockafella, RT, Wets, RJB: Variational Analysis. Grundlehren der Mathematischen Wissenschaften. Springer, Berlin (1998) 
13. Dontchev, AL, Rockafellar, RT: Implicit Functions and Solution Mappings: A View from Variational Analysis. Springer Monographs in Mathematics. Springer, Dordrecht (2009)

14. Drusvyatskiy, D, Lewis, AS: Tilt stability, uniform quadratic growth, and strong metric regularity of the subdifferential. SIAM J. Optim. 23(1), 256-267 (2013)

15. Ioffe, AD: Metric regularity and subdifferential calculus. Russ. Math. Surv. 55, 501-558 (2000)

16. Mordukhovich, BS: Variational Analysis and Generalized Differentiation. Volume I: Basic Theory. Springer, Berlin (2006)

17. Mordukhovich, BS: Variational Analysis and Generalized Differentiation. Volume II: Applications. Springer, Berlin (2006)

18. Mordukhovich, BS: Sensitivity analysis in nonsmooth optimization. In: Field, DA Komkov, V (eds.) Theoretical Aspects of Industrial Design. SIAM Proc. Applied Math., vol. 58, pp. 32-46 (1992)

19. Poliquin, RA, Rockafellar, RT: Second-Order Nonsmooth Analysis in Nonlinear Programming. World Scientific, Singapore (1995)

20. Poliquin, RA, Rockafellar, RT: Generalized Hessian properties of regularized nonsmooth functions. SIAM J. Optim. 6, 1121-1137 (1996)

21. Poliquin, RA, Rockafellar, RT: Prox-regular functions in variational analysis. Trans. Am. Math. Soc. 348(5), 1805-1838 (1996)

22. Drusvyatskiy, D, loffe, AD: Quadratic growth and critical point stability of semi-algebraic functions. Math. Program. 153(2), 635-653 (2015)

23. Wang, JJ, Song, W: Characterization of the strong metric subregularity of the Mordukhovich subdifferential on Asplund spaces. Abstr. Appl. Anal. 2014, Article ID 596582 (2014)

\section{Submit your manuscript to a SpringerOpen ${ }^{\circ}$ journal and benefit from:}

- Convenient online submission

Rigorous peer review

- Immediate publication on acceptance

- Open access: articles freely available online

- High visibility within the field

- Retaining the copyright to your article 